\title{
Activation markers of peripheral blood mononuclear cells in late pregnancy and after delivery: a pilot study
}

\author{
M Østensen, P Sicher, F Förger, P M Villiger
}

Ann Rheum Dis 2005;64:318-320. doi: 10.1136/ard.2004.022558

\begin{abstract}
Objective: To study the putative shift of a Th1 to a Th2 immune response in pregnancy and its reversal post partum in healthy women and patients with rheumatoid arthritis (RA). Methods: Peripheral blood mononuclear cells (PBMC) were examined by FACS analysis for the expression of activation markers CD25 and HLA-DR and chemokine receptors CXCR3 and CCR4 on CD4+ and CD8+ T cells in four healthy women and four patients with RA. Samples were analysed once in the third trimester and six and 12 weeks post partum. Eight healthy non-pregnant women served as controls.

Results: No reduction of CD25 and HLA-DR+ T cells occurred in the third trimester, but a significant increase was observed post partum in healthy women and an even greater increase in patients. Proportions of T cells expressing the CXCR3 or CCR4 marker were similar in patients and controls during pregnancy, whereas a significant increase occurred post partum. The ratio of CXCR3+ to CCR4+ cells remained unchanged during the observation period and did not differ significantly from that in non-pregnant controls.

Conclusion: A shift from a Th1 to a Th2 immune response was not detected in the circulation of healthy pregnant women or pregnant patients. The significant increase of T cell activation after pregnancy warrants further investigation into the mechanisms of adjustment of the immune system post partum and its clinical correlates in rheumatic patients.
\end{abstract}

$\mathrm{P}$ regnancy induces changes in the maternal immune system in order to protect the fetus from immunological attack. No general immunosuppression takes place during pregnancy, rather a shift from a Thl to a Th2 immune response has been postulated. ${ }^{1}$ This shift is of interest in diseases with a predominant Thl response and a change of disease course during pregnancy like rheumatoid arthritis (RA). ${ }^{2}$

Th1 and Th2 type $\mathrm{T}$ cells, originally defined by their cytokine profile, express a number of surface molecules which enable site-specific recruitment and effector functions. ${ }^{3}$ Thl cells express high levels of chemokine receptors CXCR3 and CCR5, whereas Th2 committed cells express CCR3, CCR4, and CCR8. ${ }^{4}$ In inflammatory rheumatic disease, increased percentages of $\mathrm{T}$ cells expressing chemokine receptors have been found in the inflamed synovium and, to a lesser degree, in peripheral blood. ${ }^{56}$ Predominant expression of CXCR3 and CCR5, but not of CCR4, has been demonstrated in active RA and juvenile chronic arthritis, indicating a predominant Thl response in RA and juvenile chronic arthritis. ${ }^{56}$

In this pilot study, healthy pregnant women and women with RA were studied in the last trimester of pregnancy and twice post partum by flow cytometric analysis to evaluate activation markers CD25 (interleukin 2 (IL2) receptor) and
HLA-DR, and chemokine receptors of CD4+ and CD8+ T cells with a focus on the Th1 and Th2 balance during pregnancy and post partum.

\section{PATIENTS AND METHODS}

Four healthy pregnant women, four pregnant patients with RA, and eight non-pregnant, healthy women were included in the study after informed consent and approval of the local ethical committee. One patient with RA received $1 \mathrm{~g}$ sulfasalazine/day throughout the study period, one patient had a non-steroidal anti-inflammatory drug at 6 weeks post partum, the other patients had no drug treatment at any time. Blood samples were drawn once in the third trimester (gestational weeks 33-39) and 6 and 12 weeks post partum.

\section{Isolation of peripheral blood mononuclear cells (PBMC)}

Venous blood was drawn into vacutainer tubes containing heparin. Peripheral blood mononuclear cells (PBMC) were isolated by the standard Ficoll-Hypaque method (Biocoll separating solution, Oxoid AG, Basel, Switzerland) within 60 minutes of collection, washed twice with phosphate buffered saline (PBS), and resuspended in PBS.

\section{Flow cytometric analysis}

Fluorescein isothiocyanate (FITC), phycoerythrin (PE), peridinin chlorophyll protein (PerCP) and allophycocyanin (APC) conjugated antihuman monoclonal antibodies (all from BD Bioscience) were used for FACS analysis: CD3 (PerCP), CD4 (FITC), CD8 (PE), IL2 receptor CD25 (APC), HLA-DR (APC), and CXCR3 (APC). Isotype matched IgG was used for control stainings: IgG2 isotype for HLA-DR, IgG1 isotype for CXCR3 and CD25, biotin mouse IgGl for CCR4.

For FACS analysis, cells were washed in staining buffer (PBS, pH 7.2 supplemented with $2 \%$ fetal calf serum and $0.1 \%$ sodium azide), incubated for 30 minutes on ice in the dark with the recommended volume of each monoclonal antibody, then washed twice with staining buffer. For the analysis of CCR4, an additional cycle of staining (the first incubation with CCR4 or IgGl biotinylated, the second with CD3/4/8) was added. Immunofluorescence and four colour flow cytometric analysis were performed using a FACS Coulter (Becton Dickinson, Mountain, View, CA) with computer interfacing to CellQuest software. The gate was set around the lymphocytes to exclude other cells from analysis. Routinely 20000 cells per tube were counted. The isotype IgG control was used for background control. The results were expressed as percentage of total PBMC in suspension, corrected for non-specific binding by antibody controls.

Abbreviations: IL, interleukin; PBMC, peripheral blood mononuclear cells; PBS, phosphate buffered saline; RA, rheumatoid arthritis 

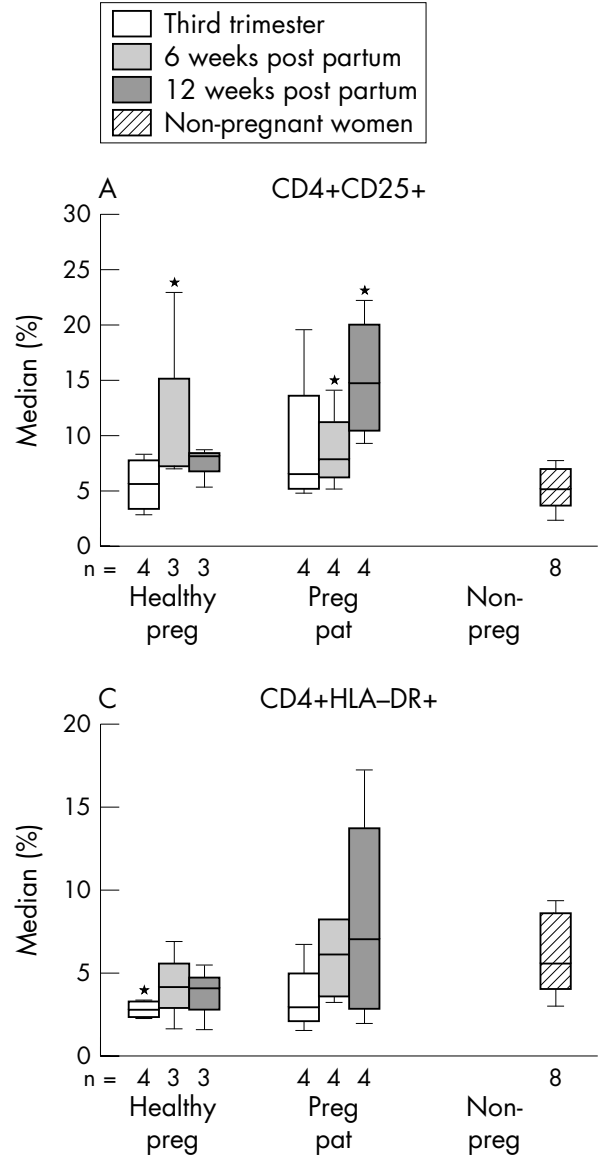

Statistics

Patients and controls were compared by the Mann-Whitney test. For comparison of third trimester and post partum samples, the Wilcoxon test was applied. A p value $\leqslant 0.05$ was regarded as significant.

\section{RESULTS}

The percentages of CD3, CD4, and CD8 positive cells did not differ significantly between pregnant and non-pregnant women (not shown). CD4+ and CD8+ $\mathrm{T}$ cells differed in their expression of CD25 and HLA-DR (fig 1). The proportion of CD25 and HLA-DR positive CD4+ and CD8+ cells in the third trimester was comparable to that in non-pregnant controls, but increased significantly post partum in healthy women and even more in patients (fig 1).

Larger numbers of CD8+ T cells were positive for CXCR3 and, conversely, lower numbers were positive for CCR4 compared with CD4+ T cells (fig 2). In the third trimester, proportions of T cells expressing the CXCR3 or CCR4 marker were similar in all groups of women, whereas a significant increase occurred post partum both in healthy women and patients compared with non-pregnant controls (fig 2). Overall, a continuous increase of cells positive for the four surface markers post partum distinguished patients from healthy pregnant women who peaked at 6 weeks post partum and then decreased. The ratio of CXCR3:CCR4 did not change significantly during the observation period and did not differ from that of non-pregnant controls (not shown).

\section{DISCUSSION}

Late pregnancy and post partum are associated with profound changes of neuroendocrine function, including
Figure 1 Percentages of CD4+ and CD8+ cells expressing activation markers CD25 and HLA-DR in four healthy pregnant women, four pregnant patients with RA, and eight healthy, non-pregnant women shown as median and range. The time of FACS analysis is shown by the key. preg, pregnant; pat, patients. *Significant difference compared with non-pregnant controls ( $p<0.05$ by Mann-Whitney $U$ test).

gestational high levels of sex steroids, corticosteroids, and catecholamines, followed by their rapid decrease after delivery. In concert with estrogens and progesterone, corticosteroids induce a Th2 response in T cells. ${ }^{7}$ In addition, they influence both $\mathrm{T}$ cell proliferation, trafficking, and function. In this pilot study of four patients with RA and four healthy pregnant women, expression of CXCR3 and CCR4 showed a similar pattern, but no predominance of the Th2 marker CCR4 in circulating $\mathrm{T}$ cells in late pregnancy. As we studied no pre-pregnancy or early pregnancy times, a gestational shift from a Th1 to a Th2 response cannot be excluded. In a study comparing 10 first trimester pregnant women with non-pregnant women, the chemokine marker CCR4 was found to be significantly raised on CD4+ T cells, but not on CD8+ $\mathrm{T}$ cells both in peripheral blood and deciduas. ${ }^{8}$ This implies that a Th2 profile may be expressed at the maternal-fetal interface or locally at a site of synovial inflammation. A recent study of the Thl/Th2 profile in pregnancy found increased IL10 mRNA expression and IL10 secretion in PBMC of four patients with RA. ${ }^{9}$ Thus, other Th2 markers not measured in this pilot study might have shown a shift from Thl to Th2 during pregnancy and a reversal post partum.

Of interest, the proportion of T cells expressing CD25, HLA$\mathrm{DR}$, or the two chemokine receptors was not lowered in the third trimester compared with non-pregnant women. Suppression of T cell activation during pregnancy (as shown by diminished proportions of CD25+ and HLA-DR+ T cells) has been observed in two longitudinal studies of healthy pregnant women. ${ }^{10}{ }^{11}$ The opposite result—namely, a higher proportion of CD25+ and HLA-DR+ T cells, was observed in two cross sectional studies comparing healthy third trimester women with non-pregnant women. ${ }^{12}{ }^{13}$ Thus a controversy 

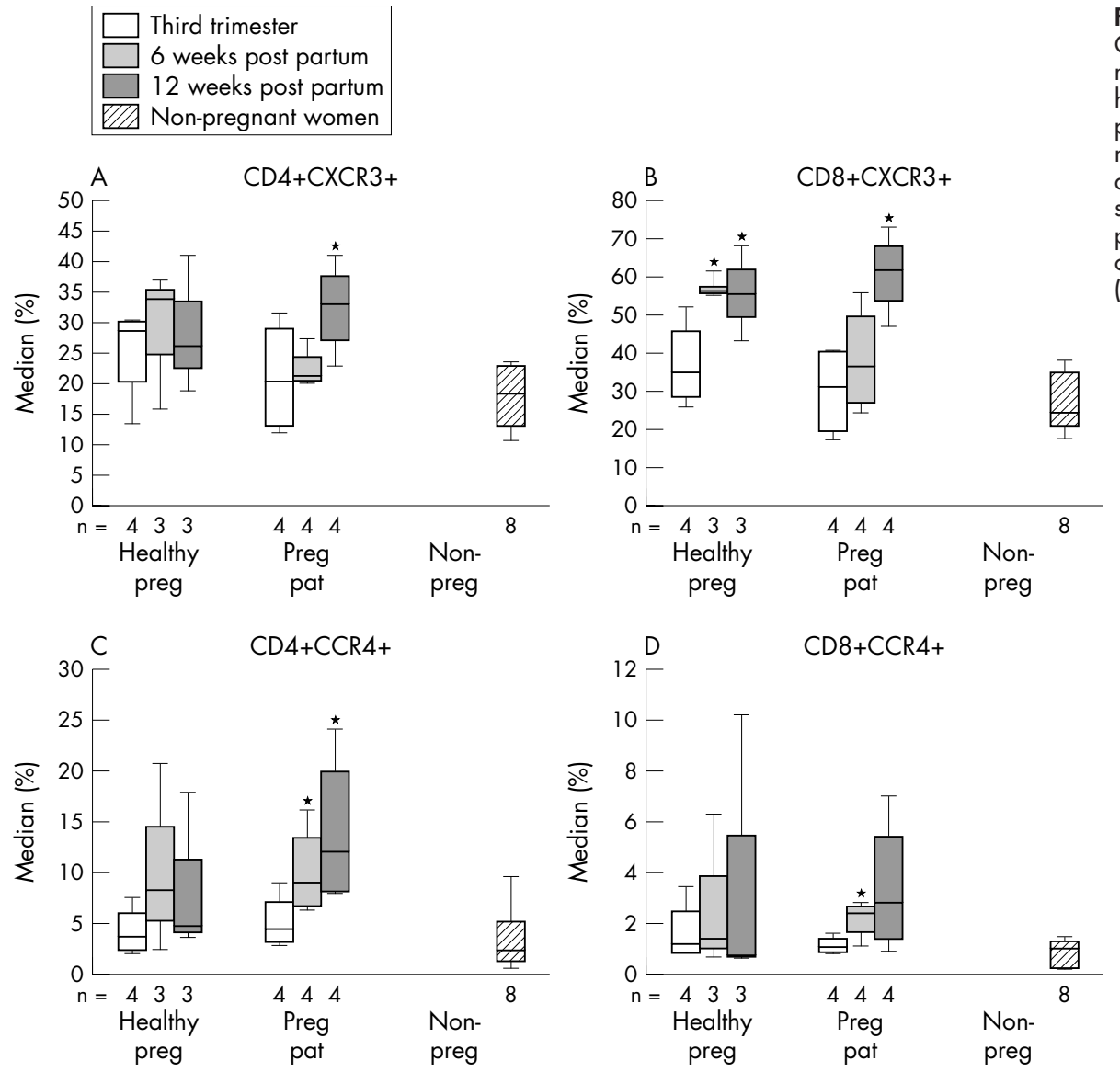

Figure 2 Percentages of $\mathrm{CD} 4+$ and CD8+ cells expressing chemokine markers CXCR3 and CCR4 in four healthy pregnant women, four pregnant patients with RA, and eight healthy, non-pregnant women shown as median and range. The time of FACS analysis is shown by the key. preg, pregnant; pat, patients. *Significant difference compared with non-pregnant controls ( $p<0.05$ by Mann-Whitney $U$ test). exists about the expression of activation markers CD25 and HLA-DR during pregnancy.

Our results indicate no general suppression of $\mathrm{T}$ cell activation in late pregnancy, but an increase of activated $\mathrm{T}$ cells in the 12 weeks after delivery, particularly in patients with RA. This fits with the timing of disease onset and of relapse in women with RA during the early post partum period. The observation warrants further investigation with larger patient numbers into the mechanisms of adjustment of the immune system post partum and its clinical correlates in rheumatic patients.

\section{ACKNOWLEDGEMENTS}

The study was supported by grant 31-59979.00 from the Swiss National Research Fund and by a grant from the Swiss League against Rheumatism.

\section{Authors' affiliations}

M Østensen, P Sicher, F Förger, P M Villiger, Department of Rheumatology and Clinical Immunology and Allergology, University Hospital, CH-3010 Bern, Switzerland

Correspondence to: Professor M Østensen, monika.oestensen@insel.ch

Accepted 1 June 2004

\section{REFERENCES}

1 Wegman TG, Lin H, Guilbert L, Mossmann TR. Bidirectional cyłokine interactions in the maternal-fetal relationship: is successful pregnancy a Th2 phenomenon? Immunol Today 1993;14:353-6.
2 Østensen M, Fuhrer L, Mathieu R, Seitz M, Villiger PM. A prospective study of pregnant patients with rheumatoid arthritis and ankylosing spondylitis using validated instruments. Ann Rheum Dis 2004;63:1212-17.

3 Mosmann TR, Sad S. The expanding universe of T cell subsets TH1, TH2 and more. Immunol Today 1996;17:138-46.

4 Sallusto F, Lenig D, Mackay CR, Lanzavecchia A. Flexible programs of chemokine receptor expression on human polarized Thelper 1 and 2 lymphocytes. J Exp Med 1998;187:875-83.

5 Ruth JH, Rottman JB, Katschke KJ, Qin S, Wu L, LaRosa G, et al. Selective lymphocyte chemokine receptor expression in the rheumatoid joint. Arthritis Rheum 2001;44:2750-60.

6 Wedderburn LR, Robinson N, Patel A, Varsani H, Woo P. Selective recruitment of polarized T cells expressing CCR5 and CXCR3 to the inflamed joints of children with juvenile idiopathic arthritis. Arthritis Rheum 2000;43:765-74

7 Elenkov IJ, Wilder RL, Bakalov VK, Link AA, Dimitrov MA, Fisher S, et al. IL12, TNF-alpha, and hormonal changes during late pregnancy and early postpartum: implications for autoimmune disease activity during these times. $J$ Clin Endocrinol Metab 2001:86:4933-8.

8 Tsuda H, Michimata T, Hayakawa S, Tanebe K, Saqkai M, Fujimura M, et al. A Th2 chemokine, TARC, produced by trophoblasts and endometrial gland cells, regulates the infiltration of CCR4+ T lymphocytes into human deciduas at early pregnancy. Am J Reprod Immunol 2002;48:1-8.

9 Munoz-Valle JF, Vazquez-Del Mercado M, Garcia-Iglesias T, OrozcoBarocio $G$, Bernard-Medina $G$, et al. $T(H) 1 / T(H) 2$ cytokine profile, metalloprotease-9 activity and hormonal status in pregnant rheumatoid arthritis and systemic lupus erythematosus patients. Clin Exp Immunol 2003;131:377-84.

10 Matthiesen L, Berg G, Ernerudh J, Hakansson L. Lymphocyte subsets and mitogen stimulation of blood lymphocytes in normal pregnancy. Am J Reprod Immunol 1996;35:70-9.

11 Kühnert M, Strohmeier R, Stegmüller M, Halberstadt E. Changes in lymphocyte subsets during normal pregnancy. Eur J Obstet Gynecol 1998;76:147-51

12 Mahmoud F, Abul H, Omu A, Al-Rayes S, Haines D, Whaley K. Pregnancyassociated changes in peripheral blood lymphocyte subpopulations in normal Kuwaiti women. Gynecol Obstet Invest 2001;52:232-6.

13 Kuhnert M, Schmidt S. Changes in lymphocyte subsets during pregnancy and post-partum in cases of beginning eclampsia. J Perinat Med 2000;28:389-98. 